\title{
EFEITO DE FATORES BIÓTICOS NO CRESCIMENTO DE HYPNEA MUSCIFORMIS (RHODOPHYTA - GIGARTINALES)
}

\author{
Renata Perpetuo Reis ${ }^{1,2}$ \\ Marta Correa Ramos Leal ${ }^{2,3}$ \\ Yocie Yoneshigue-Valentin ${ }^{4,5}$ \\ Frederico Belluco ${ }^{3}$
}

Recebido em 10/05/2002. Aceito em 15/10/2002

\begin{abstract}
RESUMO - (Efeito de fatores bióticos no crescimento de Hypnea musciformis (Rhodophyta - Gigartinales). O melhor conhecimento da ação de fatores biológicos sobre o crescimento de Hypnea musciformis (Wulfen in Jacqu.) J. V. Lamour. torna-se um aspecto premente visando a otimização do manejo e a conservação dessa espécie de interesse comercial. Uma vez que as espécies de Sargassum C. Agardh são consideradas importantes substratos para a fixação de $H$. musciformis, durante 18 meses, foram realizadas amostragens do tipo destrutiva, em uma população natural de H. musciformis epífita sobre Sargassum spp., em três profundidades de um costão rochoso no Rio de Janeiro. Obteve-se relação positiva entre as biomassas de ambos os gêneros, além da preferência destes pelo ambiente sublitorâneo. Visto a usual presença de mesoherbívoros (Amphipoda) nos talos dessas algas, a ação da herbivoria em $H$. musciformis e $S$. cymosum var. nanum foi testada em experimento in vitro. A herbivoria foi confirmada para ambos os táxons e a maior taxa de crescimento de $H$. musciformis favoreceu o crescimento de S. cymosum var.nanum, diminuindo o ataque de mesoherbívoros por ser alimento disponível. Observou-se também que não houve inibição do crescimento de H. musciformis por S. cymosum var.nanum. Sendo assim, recomenda-se que a colheita de H. musciformis para fins comerciais em bancos naturais de Sargassum spp. seja manejada para não causar danos às comunidades marinhas bentônicas.
\end{abstract}

Palavras-chave - Hypnea musciformis, relação epífita-hospedeiro, Sargassum spp., Amphypoda, herbivoria

ABSTRACT - (Effect of biotic factors on growth of Hypnea musciformis (Rhodophyta - Gigartinales). The better knowledge about the influence of biotic factors on the growth of Hypnea musciformis (Wulfen in Jacqu.) J. V. Lamour., a species of commercial interest, is necessary to the orientation of its management and conservation. Since the specimens of Sargassum C. Agardh are considered an important substrate for the fixation of H. musciformis, destructive sampling were done during eighteen months in natural population of $H$. musciformis

1 Instituto de Pesquisas Jardim Botânico do Rio de Janeiro, Rua Pacheco Leão 915, CEP 22460-030, Rio de Janeiro, RJ, Brasil

2 Auxílio Pesquisa da FAPERJ

3 Bolsista PIBIC/CNPq

4 Universidade Federal do Rio de Janeiro, Instituto de Biologia, Departamento de Botânica

5 Pós Graduação em Biotecnologia Vegetal, Centro de Ciências da Saúde, Ilha do Fundão, CEP 21941-900, Rio de Janeiro, RJ, Brasil

Autor para correspondência: rreis@jbrj.gov.br 
growing on Sargassum spp. in three deepths on the rocky shore at Rio de Janeiro. A positive correlation was obtained for the biomass of both genus and the preference for subtidal habitat was observed. Amphipods are usually found in their frond so the impact of mesograzers (amphipods) in H. musciformis and S. cymosum var. nanum were tested in experiments in vitro. The amphipods grazing was confirmed for both species and $H$. musciformis, that has higher growth rate, favored the growth of S. cymosum var.nanum, reducing the mesograzers attack by being available food. So, we recommend that commercial harvest of $H$. musciformis growing on Sargassum spp. should be managed to avoid damage to the marine ecosystem.

Key words - Hypnea musciformis, Sargassum spp., Amphypoda, herbivore

\section{Introdução}

Existem diferentes interpretações sobre os vários tipos de relação entre a epífita e o hospedeiro em macroalgas, assim como a ação da herbivoria sobre ambos. Alguns autores sugerem que a maioria das rodofíceas, quando epífitas, usa o hospedeiro meramente como substrato (Kain \& Norton 1990), enquanto outros citam a interferência das epífitas no crescimento do hospedeiro, como um atenuante da intensidade luminosa devido à sua biomassa sobre o hospedeiro (Buschmann \& Gomez 1993). Além disso, a herbivoria tem sido reconhecida como fator determinante na relação epífita-hospedeiro, ou aumentando o estabelecimento da macroalga, como conseqüência da eliminação das epífitas por anfípodas que competiriam com a hospedeira (Duffy 1990), fato este observado com espécies de Hypnea (Brawley \& Adey 1981), ou, de modo contrário, o impacto negativo na biomassa da alga causado pela intensa herbivoria (Duffy 1990).

Na relação epífita-hospedeira existe uma rede complexa de benefícios e desvantagens na qual a importância relativa de efeitos positivos e negativos é determinada, em cada caso, por inúmeros fatores ambientais e espécieespecíficos (Wahl 1989).

Nos ecossistemas marinhos da zona costeira brasileira, tem sido observado que populações de Hypnea musciformis (Wulfen in Jacqu.) J. V. Lamour. utilizam indivíduos de Sargassum C. Agardh como importante substrato para o seu desenvolvimento (Schenkman 1989; Berchez
1990; Berchez et al. 1993; Reis \& YoneshigueValentin 1998). Tem sido constatada também, a presença maciça de herbívoros (anfípodas) em bancos naturais de H. musciformis no Estado do Rio de Janeiro (observação pessoal) e no Estado de São Paulo (Schenkman 1989), como também em ensaios de maricultura com H. musciformis no estado de São Paulo (Berchez 1990).

Hypnea musciformis é uma espécie de interesse comercial devido à presença de kapa carragenana em sua parede celular. Esta substância é utilizada industrialmente como agente geleificante, estabilizante, espessante ou emulsificante (Reis 1998). Esta espécie vem sendo explorada por décadas como matéria prima para produção de carragenana, porém sem estimativa de seus estoques naturais na costa brasileira (Oliveira 1998).

Sendo assim, devido à necessidade de ampliar-se o conhecimento sobre populações de algas de interesse comercial, avaliou-se, durante 18 meses, a relação entre a biomassa de uma população de Hypnea musciformis epífita sobre a biomassa de Sargassum spp. em um local da baía de Sepetiba. Além disso, o efeito da herbivoria por anfípodas sobre as duas espécies, foi testado experimentalmente in vitro.

\section{Material e métodos}

Amostragem in situ - Amostras de biomassa de Hypnea musciformis e de Sargassum spp. foram obtidas bimestralmente na praia Grande, na ilha de Itacuruçá, baía de Sepetiba, município de Mangaratiba, Estado do 
Rio de Janeiro ( $22^{\circ} 57^{\prime} \mathrm{S}$ e $43^{\circ} 54^{\prime} \mathrm{W}$ ) entre o período de outubro/1994 a abril/1996, através da utilização de quadrados de $625 \mathrm{~cm}^{2}$, tamanho este normalmente utilizado em estudos de populações monoespecíficas com talo de pequeno porte, como é o caso de H. musciformis (Chapman 1985; De Wreede 1985). Neste local foi encontrado denso e extenso banco de H. musciformis como epífita de Sargassum spp. As dinâmicas sazonais de $H$. musciformis e de Sargassum spp. foram avaliadas através das medidas de biomassa seca (obtidas em estufa a $60{ }^{\circ} \mathrm{C}$ até a obtenção de massa constante). A determinação do número mínimo de amostras (quadrados) necessárias à estimativa das médias das populações foi obtida através das representações gráficas das médias acumuladas das biomassas, em massa seca de H. musciformis e de Sargassum spp. em relação ao número acumulado de amostras (Chapman 1985). Estimou-se em sete o número de quadrados suficientes para a amostragem das populações de ambos os gêneros (Reis 1998).

Os quadrados foram dispostos aleatoriamente sobre três transectos horizontais de 20 metros de extensão, em três profundidades no costão rochoso (inclinação média de $40^{\circ}$ ), na faixa de ocorrência da população de H. musciformis: a) no limite superior da faixa de $H$. musciformis, $0,1 \mathrm{~m}$ acima do nível médio das baixa-mares de sizígia; b) na zona intermediária correspondendo à $0,1 \mathrm{~m}$ abaixo do nível médio das baixa-mares de sizígia e c) no limite inferior do habitat de H. musciformis, localizado à $0,4 \mathrm{~m}$ abaixo do nível médio das baixa-mares de sizígia. As biomassas secas de H. musciformis e de Sargassum spp. contidas nos 210 quadrados foram analisadas seguindo a metodologia de amostragem do tipo destrutiva proposta por De Wreede (1985).

Experimentos in vitro - As condições de cultivo foram as seguintes: temperatura ambiente $22 \pm 2{ }^{\circ} \mathrm{C}$, intensidade luminosa de $50 \pm 5 \mu \mathrm{mol}$ fótons. $\mathrm{m}^{-2} \cdot \mathrm{s}^{-1}$ na superfície da água, fotoperíodo de 12 horas de luz por dia e salinidade da água do mar próxima a 35 . O meio de cultura foi constituído por água do mar filtrada $(0,45 \mu \mathrm{m}$ de poro $)$ e esterilizada em forno de microondas (1 litro por 5 min na potência máxima), ao qual foi adicionado dióxido de germânio $\left(\mathrm{Ge}_{2} \mathrm{O}_{2}\right)$ e penicilina. Penicilina e $\mathrm{Ge}_{2} \mathrm{O}_{2}$ foram usados para excluir os contaminantes, como as bactérias e as diatomáceas (Stein 1973). O experimento teve duração de sete dias.

Exemplares de H. musciformis e de Sargassum cymosum var. nanum E. de Paula foram coletados na Praia Rasa, no município de Armação de Búzios, Estado do Rio de Janeiro (22 $2^{\circ} 4 \mathrm{~S}^{\prime}$ e $41^{\circ} 56^{\prime} \mathrm{W}$ ), em 18/outubro/2001. Seis réplicas, com cerca de $0,25 \mathrm{~g}$ de massa úmida de alga, foram selecionadas para cada tratamento. Para assegurar a independência das amostras foram utilizadas porções apicais vegetativas de $H$. musciformis e de $S$. cymosum var. nanum, de espécimes distintos e os recipientes de vidro de cada experimento foram dispostos aleatoriamente na mesma prateleira.

$\mathrm{O}$ experimento foi realizado em frascos do tipo Erlenmeyer, com $200 \mathrm{~mL}$ de meio, com aeração constante, nos quais $H$. musciformis e Sargassum cymosum var. nanum cresceram juntos (Fig. 1a); como controle cada táxon foi cultivado isolado (Fig. 1b e 1c) e H. musciformis foi encubada com uma planta plástica que mimetizou S. cymosum var. nanum (Fig. 1d). A ação da herbivoria sobre estas duas espécies foi testada pela adição de três anfípodas em cada frasco com cada espécie (Fig. 1e e 1f) e com as duas espécies juntas (Fig. 1g) que foram comparadas com os táxons que cresceram isolados (Fig. 1b e $1 \mathrm{c}$ ).

$\mathrm{O}$ crescimento das algas foi estimado através da fórmula da Taxa de Crescimento Relativo (TCR), na qual, $R=\left(\ln \mathrm{M}_{\mathrm{t}}-\ln \mathrm{M}_{\mathrm{o}}\right) / \mathrm{T}$, onde $\mathrm{R}=$ taxa de crescimento relativo, $M_{\mathrm{o}}=$ massa seca inicial, $\mathrm{M}_{\mathrm{t}}=$ massa seca medida no dia te $\mathrm{T}$ = número de dias (Kain 1987).

A correlação entre $H$. musciformis e Sargassum spp. nas diferentes profundidades 

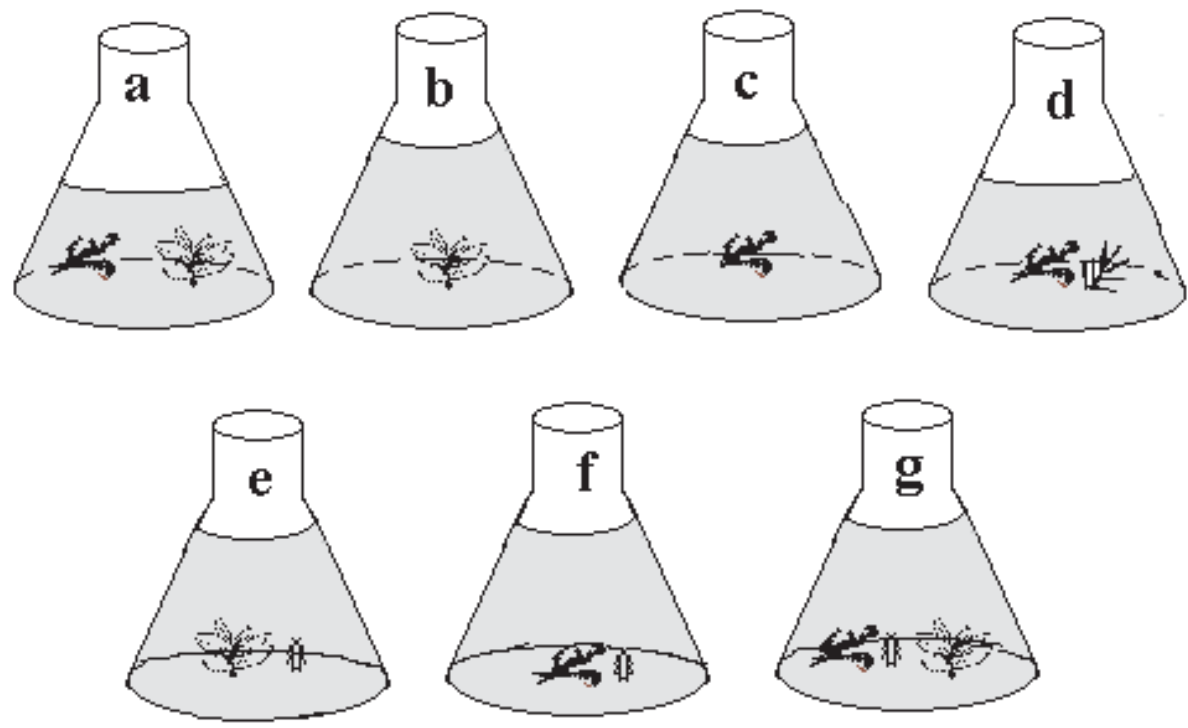

trapnea rmosciformis

然

花 anfípoda

Figura 1. Esquema do experimento in vitro com Hypnea musciformis e Sargassum cymosum var. nanum crescendo juntos (a), isolados (b e c), H. musciformis encubada com planta plástica mimetizando Sargassum (d), cada táxon crescendo junto com anfípodas (e e f) e ambos táxons juntos com três anfípodas (g).

do costão foi obtida através do coeficiente de correlação de Pearson (Zar 1996). A diferença entre os tratamentos dos ensaios in vitro foi obtida pela análise de variância (ANOVA) unifatorial e as diferenças entre as médias através do teste de Tukey (Zar 1996). Estas análises foram escolhidas por serem consideradas robustas para desvios na normalidade (Underwood 1981; Zar 1996). A homogeneidade das variâncias foi verificada através do teste de Cochran (Zar 1996; Winer 1971). O grau de confiança para todos os testes de significância foi estipulado em $95 \%$ ( $p=0.05)$. Os dados estão apresentados em média \pm desvio padrão. Os fatores foram considerados fixos e independentes, de acordo com as recomendações de Underwood (1981) e Zar (1996).

\section{Resultados e discussão}

A amostragem das biomassas de H. musciformis e de Sargassum spp.
(S. cymosum var. cymosum e $S$. filipendula var. filipendula) obtidas em campo, nas três profundidades num período de 18 meses, resultou em correlação positiva $r=0,65, p<0,001, n=$ 210. A Fig. 2 retrata as variações sazonais das biomassas de H. musciformis e de Sargassum spp., nos três níveis do costão da praia Grande. Nela observa-se a tendência geral da correlação entre esses dois gêneros, como no período de agosto/1995 a abril/1996, quando a biomassa de Sargassum spp. decresceu junto com a de $H$. musciformis. Outro momento que retrata bem esta relação positiva foi em outubro/1994, no nível superior do costão, quando ocorreram as maiores biomassas de Sargassum spp. e de H. musciformis, evidenciando a utilização de Sargassum spp. como substrato disponível para a colonização de $H$. musciformis. Resultados semelhantes foram obtidos em populações naturais de $S$. cymosum com $H$. musciformis como epífita no Estado de São Paulo (Schenkman 1989). 

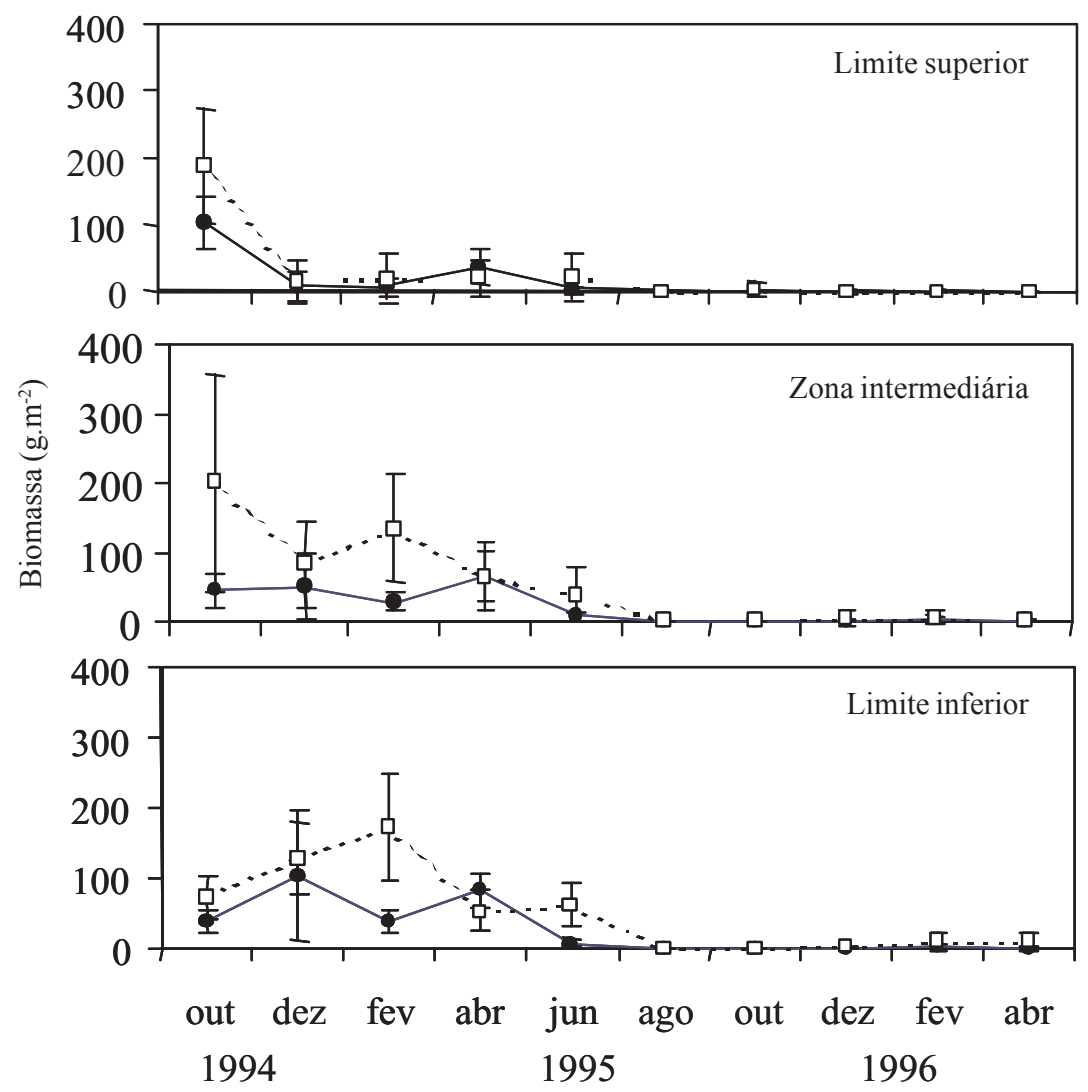

Figura 2. Variação temporal das biomassas (massa seca) de Hypnea musciformis e Sargassum spp., no limite superior da faixa de $H$. musciformis, $0,1 \mathrm{~m}$ acima do nível médio das baixa-mares de sizígia; na zona intermediária correspondendo à $0,1 \mathrm{~m}$ abaixo do nível médio das baixa-mares de sizígia e no limite inferior do habitat de H. musciformisno no costão rochoso da Praia Grande, Itacuruçá, RJ. Quadrado aberto representa a média dos valores da biomassa de Sargassum (S. cymosum var. cymosum e $S$. filipendula var. filipendula) e circulo fechado de H. musciformis e a linha o desvio padrão (ANOVA, $\mathrm{p}<0,001, \mathrm{n}=7$ ). $-\bullet-$ Hypnea musciformis; --- $\square---$ Sargassum.

As maiores biomassas, tanto do hospedeiro quanto da epífita, ocorreram no nível inferior do costão (zona intermediária e limite inferior) comprovando a preferência desses táxons pelo habitat sublitorâneo. No limite superior, a biomassa de $H$. musciformis foi de 15,82 \pm $34,63 \mathrm{~g} \cdot \mathrm{m}^{-2}$, na zona intermediária de $33,74 \pm$ $49,16 \mathrm{~g} \cdot \mathrm{m}^{-2}$, e no limite inferior foi de $34,72 \pm$ $33,08 \mathrm{~g} \cdot \mathrm{m}^{-2} \mathrm{e}$ as de Sargassum spp. foram de $26,72 \pm 63,19 \mathrm{~g} \cdot \mathrm{m}^{-2}$ no limite superior, 63,02 \pm $67,34 \mathrm{~g} \cdot \mathrm{m}^{-2}$ na zona intermediária e $66,08 \pm$ 93,01 g.m $\mathrm{m}^{-2}$ no limite inferior.

No experimento in vitro (Fig. 1) idealizado para averiguar o impacto da herbivoria sobre Hypnea musciformis e Sargassum cymosum var. nanum e verificar a existência de propriedades antibióticas associadas às espécies de Sargassum, gênero conhecido por produzir substâncias de defesa frente a herbívoros ou alelopáticas (Sieburth \& Conover 1965; Pereira \& Yoneshigue-Valentin 1999), constatou-se o impacto da herbivoria por anfípodas em H. musciformis como pode ser observado na Fig. 3, pelo menor crescimento desta espécie na presença de anfípodas (tratamento $\mathrm{H}$ com tratamento $\mathrm{H}+\mathrm{A}$, ANOVA, $\mathrm{F}=9,91, \mathrm{p}=0,01$ ). 


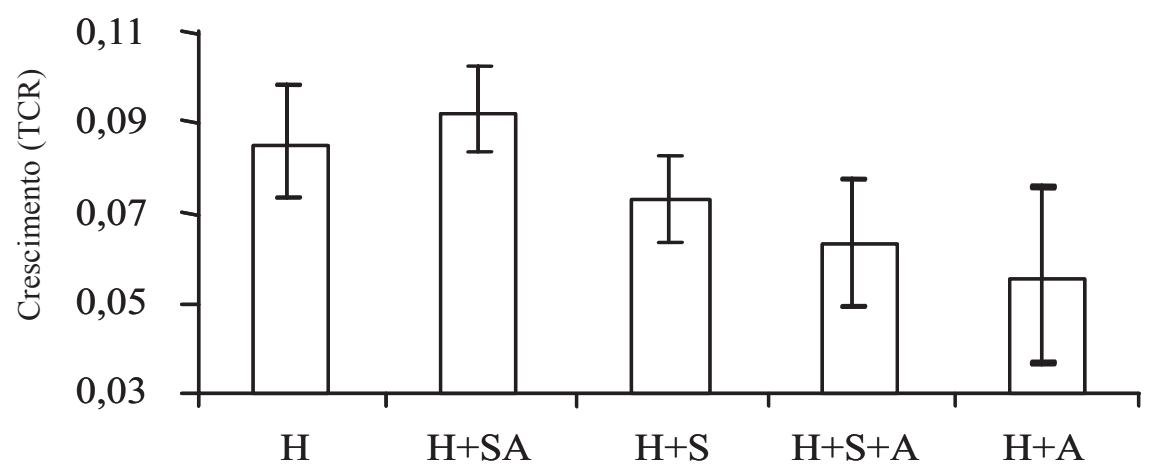

Figura 3. Crescimento (Taxa de Crescimento Relativo) de Hypnea musciformis cultivada in vitro, em Erlenmeyer, crescendo isolada $(\mathrm{H})$, sobre substrato artificial $(\mathrm{H}+\mathrm{SA})$, sobre Sargassum cymosum var. nanum $(\mathrm{H}+\mathrm{S})$, sobre S. cymosum var. nanum e mais três anfípodas $(\mathrm{H}+\mathrm{S}+\mathrm{A})$ e com três anfípodas $(\mathrm{H}+\mathrm{A})$. A linha em cada barra de valor médio indica o desvio padrão $(\mathrm{n}=6)$.

O mesmo ocorreu com $S$. cymosum var. nanum, quando observada a Fig. 4, (tratamento S com tratamento $\mathrm{S}+\mathrm{A}, \mathrm{ANOVA}, \mathrm{F}=64,39, \mathrm{p}<0,001$, $\mathrm{n}=6$ ), corroborando os resultados de ensaios de maricultura com $H$. musciformis no Estado de São Paulo, assim como as observações sobre as populações naturais desta espécie no estado de São Paulo (Schenkman 1989; Berchez 1990) e do Rio de Janeiro (observação pessoal).

O crescimento de Hypnea musciformis não foi inibido pela presença de $S$. cymosum var. nanum (Fig. 3), visto que não houve diferença entre os tratamentos de $H$. musciformis crescendo isolada com espécimes crescendo sobre S. cymosum var. nanum (tratamento $\mathrm{H}$ com tratamento $\mathrm{H}+\mathrm{S}$, ANOVA, $\mathrm{F}=3,76, \mathrm{p}=$ $0,08, \mathrm{n}=6$ ). Isso ocorreu, provavelmente, devido à maior taxa de crescimento de $H$. musciformis em comparação com a taxa de $S$. cymosum var. nanum (tratamento $\mathrm{H}$ com tratamento $\mathrm{S}$, ANOVA, $\mathrm{F}=130,65, \mathrm{p}<0,001, \mathrm{n}=6$, Fig. 3 e 4). A taxa de crescimento de $H$. musciformis foi de $0,09 \pm 0,01$ e a de $S$. cymosum var. nanum $0,02 \pm 0,01$, ou seja, uma taxa de crescimento quase cinco vezes maior, comprovando assim ser uma espécie de rápido crescimento, concordando com os comentários de Kain (1987) e, por isto, como recomendados por Schenckman (1989), Berchez (1990) e Reis (1998) justificando seu potencial para maricultura.

$\mathrm{Na}$ presença de anfípodas, Sargassum cymosum var. nanum sempre apresentou menor crescimento (Fig. 4). Por outro lado, ao comparar o tratamento desta espécie encubado com $H$. musciformis e anfípodas (tratamento $\mathrm{S}+\mathrm{H}+\mathrm{A}$ ) com o tratamento com anfípodas e na ausência de $H$. musciformis (tratamento $\mathrm{S}+\mathrm{A}$ ) o crescimento foi significativamente maior na presença de $H$. musciformis (ANOVA, $\mathrm{F}=16,40$ $\mathrm{p}=0,002, \mathrm{n}=6$ ). Isto comprova que esta alga favorece o crescimento de $S$. cymosum var. nanum através da minimização do ataque de mesoherbívoros, devido à taxa de crescimento que é maior do que a de $S$. cymosum var. nanum, atuando desta forma, como alimento disponível para os mesoherbívoros.

Concluindo, observou-se a preferência de H. musciformis, Sargassm cymosum var. cymosum e $S$. filipendula var. filipendula pelo ambiente sublitorâneo e que o aumento da biomassa de $H$. musciformis relacionou-se ao aumento da biomassa dessas espécies, como substrato disponível. Não houve inibição do crescimento de $H$. musciformis por $S$. cymosum var. nanum e na presença de anfípodas o crescimento desta espécie foi favorecido pela presença de $H$. musciformis. Em uma semana de cultivo in vitro, estas espécies não 


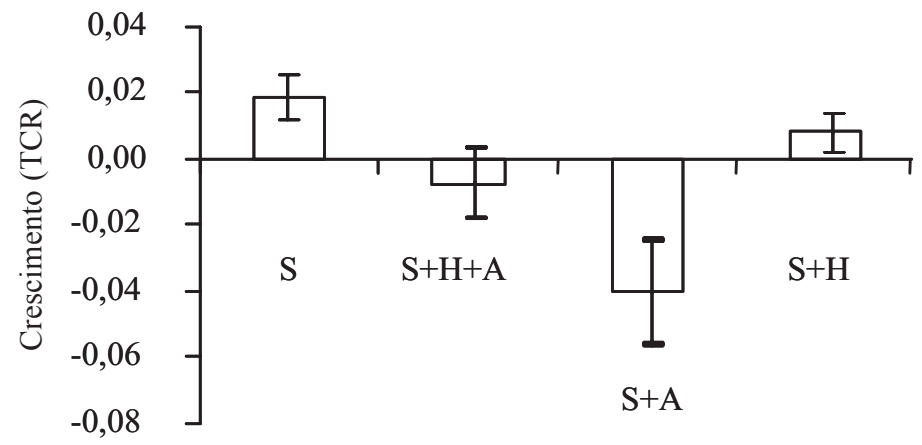

Figura 4. Crescimento (Taxa de Crescimento Relativo) de Sargassum cymosum var. nanum cultivado in vitro, em Erlenmeyer, crescendo isolado (S), com Hypnea musciformis e três anfípodas $(\mathrm{S}+\mathrm{H}+\mathrm{A})$, com três anfípodas $(\mathrm{S}+\mathrm{A})$ e com Hypnea musciformis $(\mathrm{S}+\mathrm{H})$. A linha em cada barra de valor médio indica o desvio padrão $(\mathrm{n}=6)$.

produziram defesa química contra herbivoria. Foi também comprovada a alta taxa de crescimento de H. musciformis.

Recomenda-se que a colheita de Hypnea musciformis para fins comerciais em bancos naturais de Sargassum spp. seja manejada, para não causar danos ao hospedeiro e conseqüentemente às comunidades marinhas bentônicas.

\section{Agradecimentos}

À Fundação de Amparo à Pesquisa do Estado do Rio de Janeiro - FAPERJ (Processo E 26/171.610/1999), pelo auxílio financeiro; ao Programa Institucional de Iniciação Científica do Conselho Nacional de Desenvolvimento Científico e Tecnológico - PIBIC/CNPq; ao Dr Renato Crespo Pereira, pelas críticas e sugestões.

\section{Referências bibliográficas}

Berchez, F. A. S. 1990. Ensaios de maricultura da alga Hypnea musciformis (Rhodophyta, Gigartinales) no litoral do Estado de São Paulo. Tese de Doutorado. Universidade de São Paulo, São Paulo.

Berchez, F. A. S.; Pereira, R. T. L. \& Kamiya, N. F. 1993. Culture of Hypnea musciformis (Rhodophyta - Gigartinales) on artificial substrates attached to linear ropes. Hydrobiologia 260/261: 415-420.
Brawley, S. H. \& Adey, W. H. 1981. Micrograzers may affect macroalgal density. Nature 292: 177.

Buschmann, A. H. \& Gomez, P. 1993. Interaction mechanisms between Gracilaria chilensis (Rhodophyta) and ephiphytes. Hydrobiologia 260/261: 743-813.

Chapman, A.R.O. 1985. Demography. Pp. 251-268. In M.M. Littler \& D. S. Littler (ed.) Handbook of Phycological Methods Ecological Field Methods: Macroalgae. Cambridge University Press, New York.

De Wreede, R.E. 1985. Destructive (harvest) sampling. Pp. 147-160. In M.M. Littler \& D. S. Littler (ed.) Handbook of Phycological Methods Ecological Field Methods: Macroalgae. Cambridge University Press, New York.

Duffy, J. E. 1990. Amphipods on seaweeds: partners or pest? Oecologia 83: 267-276.

Kain, J. M. 1987. Seasonal growth and photohibition in Plocamium cartilagineum (Rhodophyta) of the Isle of Man Phycologia 26(1):88-99.

Kain, J. M. \& Norton, T. A. 1990. Marine Ecology. Pp. 377-422. In: Cole K. M. \& R. G. Sheath (eds.) Biology of the red algae. Cambridge University Press, New York.

Oliveira, E. C. 1998. The seaweed resourses of Brazil. Pp. 366-371. In: Critchley, A. T. \& M. Ohno (eds.) Seaweeds Resources of the Wold. Japan International Cooperation Agency.

Pereira, R. C. \& Yoneshigue-Valentin, Y. 1999. The role of polyphenols from the brown alga Sargassum furcatum on the feeding by amphipod herbivores. Botanica Marina 42: 441-448. 
Reis, R. P. 1998. Variação espaço-temporal de carragenana em Hypnea musciformis (Wulfen) Lamouroux (Rhodophyta - Gigartinales) em bancos naturais do Estado do Rio de Janeiro, Brasil. Tese de Doutorado. Universidade Federal do Rio de Janeiro, Rio de Janeiro.

Reis, R. P. \& Yoneshigue-Valentin, Y. 1998. Variação espaço-temporal de populações de Hypnea musciformis (Rhodophyta, Gigartinales) na Baía de Sepetiba e Armação dos Búzios, Rio de Janeiro, Brasil. Acta Botanica Brasilica 13(2): 465-83

Schenkman, R. P. F. 1989. Hypnea musciformis (Rhodophyta): ecological influence on growth. Journal of Phycology 25: 192-196.

Sieburth, J. Mc. N \& Conover, J. T. 1965. Sargassum tannin, an antibiotic which retards fouling. Nature 208: 52-53.
Stein, J. R. 1973. Handbook of phycological methods culture methods and growth measurements. Cambridge University Press.

Underwood, A. J. 1981. Techniques of analysis of variance in experimental marine biology and ecology. Oceanography and Marine Biology Annual Review 19: 513-605.

Wahl, M. 1989. Marine epibiosis. I. Fouling and antifouling: some basic aspects. Marine Ecology Progress Series 58: 175-189.

Winer, B. J. 1971. Statistical principles in experimental design. Second editions. McGrawHill, Tokyo.

Zar, J. H. 1996. Biostatistical analysis. Third editions Prentice-Hall International Editions, New Jersey. 